\title{
Failure to find disease similarity in sibling pairs with rheumatoid arthritis
}

\author{
ALAN J SILMAN, W E R OLLIER, AND H L F CURREY \\ From the Departments of Clinical Epidemiology and Tissue Immunology and the Bone and Joint Research \\ Unit, London Hospital Medical College, London
}

SUMMARY Clinical and laboratory measures of disease expression were compared within and between 33 families with two or more affected siblings with rheumatoid arthritis (RA). None of the variables studied-age and calendar year of disease onset, pattern of joint involvement, the presence of rheumatoid nodules, Sjögren's syndrome, a positive latex or antinuclear antibody (ANA) titre-showed a greater concordance within the families than between them. The families were then divided into those in which the affected sibling pairs were and were not HLA identical. Such a division did not alter the conclusion, with the possible exception of a positive latex titre. These results suggest that genetic or unique environmental factors within families may have only a limited role in explaining disease heterogeneity in RA. Conversely, the absence of homogeneity within the families justifies their use in genetic linkage studies and the extrapolation of results obtained from affected siblings to the commoner sporadic disease.

Key words: HLA, joint distribution, nodules, Sjögren's syndrome, seropositivity.

The existence of a genetic component in rheumatoid arthritis (RA) is supported by evidence of clustering of disease in families, ${ }^{1}$ with a greater concordance in monozygotic than dizygotic twins. ${ }^{2}$ Recent studies of HLA antigens in affected and non-affected unrelated individuals have shown an association between HLA-DR4/Dw4 and RA, suggesting that genetic factors may be important in sporadic cases. $^{34}$ The disease shows considerable heterogeneity in its clinical expression and one obvious explanation for this could be that the genetic component in aetiology varies between disease subgroups. Thus patients with seropositive RA are more likely to have a family history ${ }^{1}$ and be HLA-DR4 positive ${ }^{5-7}$; though the latter is not a universal finding. ${ }^{4}$ Most patients with RA have no family history, suggesting a multifactorial disease aetiology. The relative influence of genetic and environmental factors is unknown but may vary in different subgroups of RA.

The existence in a family of two or more affected members with RA suggests that a shared genetic or environmental factor, or both, may be responsible, at least in part, for their susceptibility to the disease.

Accepted for publication 7 July 1986.

Correspondence to $\mathrm{Dr}$ Alan J Silman. Department of Clinical Epidemiology. London Hospital Medical College. Turner Street, London E1 2AD.
In this report the hypothesis is tested that the pattern of disease expression within these families is shaped by the same factors determining their disease susceptibility. If the hypothesis were true similarity in certain aspects of disease expression might be expected. The absence of such observed similarity would argue against disease expression being strongly linked genetically within families. This question is of practical importance to those using multicase families as a tool for examining linkage with specific genetic markers. Greater disease homogeneity within, rather than between, affected families would suggest that conclusions about any linkage observed could not be extrapolated generally.

We have compared disease heterogeneity within and between affected members of families with multiple cases of RA. The analysis is restricted to affected siblings to exclude bias due to differences in disease duration between generations.

\section{Patients and methods}

We have investigated 27 sib pairs and six sib trios with RA seen as part of a family study. Each affected individual satisfied the standard American Rheumatism Association criteria for classical or definite RA. $^{8}$ 
Each affected individual was examined by one of two observers and the information recorded in a standardised manner using a fixed format data record sheet. The disease variables studied included both age and calendar year of disease onset as determined by recalled date of fiz $;$ symptoms. This date was checked where possible either with contemporary medical records or by interviewing a cohabiting relative, normally a spouse. Clinical measures are notoriously sensitive to change and thus, for this analysis, were mainly restricted to the identification of rheumatoid nodules and Sjögren's syndrome (using Schirmer's test). Additionally, in view of recent reports that different subgroups of patients with RA could be categorised by the radiological pattern of small joint involvement in the wrists and hands, ${ }^{9}$ each patient was examined to determine whether either carpal or metacarpophalangeal (MCP), or both joint groups, were involved.

Serum was obtained for the measurement of latex and ANA titre; all assays being performed by the same technician. Finally, all patients, and other members of the family, were HLA typed, and disease heterogeneity was compared between HLA identical and non-identical sib pairs.

A N A L YS IS

The concordance between sibling pairs for the categorical variables studied, for example nodules, could be expressed using the format below:

\begin{tabular}{llll} 
Nodules & & \multicolumn{2}{c}{ Sibling A } \\
\hline & & YES & $\begin{array}{l}\text { NO } \\
\text { Sibling B }\end{array}$ \\
& NOS & a & b \\
& c & d
\end{tabular}

Each pair could then be allocated to one of the cells ' $a$ ' to ' $d$ ' depending on the presence or absence of nodules in the two members. Cells ' $a$ ' and ' $d$ ' represent concordant pairs, i.e., both members identical in their nodule status, and cells ' $b$ ' and ' $c$ ' represent discordant pairs. A $\chi^{2}$ test could test the significance of any deviation of the observed frequency of pairs in the categories from that expected based on chance as determined by the overall prevalence of (in the example) nodules. Such a test is insufficient for the present study as it is desirable to know both the direction of any deviation from the expected distribution, i.e., towards greater concordance or greater discordance, and also the strength or likely importance of the deviation. An alternative analysis is to use Cohen's kappa, ${ }^{10}$ which was originally described to compare the agreement between two raters. Its use has been recently extended by Fleiss to assess concordance between individuals, ${ }^{11}$ as in the present study. The degree of concordance is expressed as a numerical value for kappa $(x)$ which can range from $-1 \cdot 0$, indicating perfect non-concordance, to $+1 \cdot 0$, indicating perfect concordance. A numerical value above $0.75 \stackrel{0}{?}$ probably indicates important concordance, whereas? a value below 0.45 has little meaning. A perfectly random result produces a value for $x$ of 0 . The observed value of $x$ can be tested for statistical $\mathbb{Q}$ significance $\left(\mathrm{H}_{0} x=0\right)$ by calculating $\mathrm{SE} x$, as $x / \mathrm{SE} x_{\omega}^{\infty}$ follows a $\mathrm{Z}$ distribution. Hence a $95 \%$ confidenceinterval for the calculated values of $x$ can be determined using $x \pm 1.96 \mathrm{SE} x$.

The concordance between the sibling trios could be handled separately from that between the pairs. For the dichotomous variables, whatever the preva-i lence, at least two individuals of the trio must be concordant. Thus treating a trio $(\mathrm{A}, \mathrm{B}, \mathrm{C})$ as three pairs $(A B, A C, B C)$ would perhaps weight the results towards a greater concordance. In practices the difference between the observed and expectedconcordance in the trios was similar whether analy- $T$ sed as ' $n$ ' trios or as three ' $n$ ' sibling pairs. In the을 latter case the six trios generated 18 sibling pairs, which were analysed jointly with the 27 other pairs.

Finally, the concordance within families for the $\overrightarrow{0}$ continuous variables was studied using the $F$ tes $t$ for a two way analysis of variance.

\section{Results}

Within the sibling pairs there was a mean differenced in age of RA onset of 14.3 years (SD 11.7, range $1-61)$, and the variation within pairs was noe significantly less than that between pairs $(F=0.78) \frac{3}{\sigma}$ Similarly, there was a mean difference in calendaf year of disease onset of 14.8 years (SD 13.5, range 1-59), and again the variation within pairs was no significantly less than that between pairs $(F=0.49) \div$

Each pair had at least one individual with at leas one of the two joint groups (MCP/carpus) involved and in half the pairs both joint groups were involved in both individuals (Table 1). Interestingly, thereo was only one pair in which neither individual had both joint groups involved.

Table 1 Pattern of joint involvement: involvement of carpus or MCP joints, or both

\begin{tabular}{|c|c|c|}
\hline First member & Second member & Number of pairs \\
\hline $\mathrm{MCP}+$ carpus & $\mathrm{MCP}+$ carpus & 23 \\
\hline MCP + carpus & MCP & 11 \\
\hline MCP + carpus & Carpus & 8 \\
\hline MCP + carpus & Neither & 2 \\
\hline MCP & Neither & 1 \\
\hline & & $\overline{45}$ \\
\hline
\end{tabular}


Table 2 Concordance for nodules and Sjögren's syndrome

\begin{tabular}{lll}
\hline & \multicolumn{2}{l}{ Number of pairs } \\
\cline { 2 - 3 } & Nodules & Sjögren's \\
\hline Both members & 13 & 3 \\
One member & 26 & 17 \\
Neither member & 6 & 25 \\
Kappa & -0.08 & $-0 \cdot 01$ \\
SE & $0 \cdot 19$ & $0 \cdot 15$ \\
95\% Confidence & $-0 \cdot 27$ & $-0 \cdot 30$ \\
$\quad$ interval & $+0 \cdot 11$ & $+0 \cdot 28$ \\
p Value & NS & NS \\
\hline
\end{tabular}

The agreement for nodules was poor (Table 2) $(x=-0.08$, p NS), with most pairs (26) being discordant. Similarly, Sjögren's syndrome, when present, was normally discordant between the pairs $(x=-0.01, p$ NS). Perhaps surprisingly there was no concordance for a positive latex titre $(1 / 80$ or above), with the distribution within pairs being that expected by chance $(x=0 \cdot 00$, p NS) (Table 3$)$. A similar observation was seen with ANA with a titre of $1 / 10$ or above $(x=0.04$, p NS) (Table 3$)$. For each of the above four variables the upper $95 \%$ confidence limit was below $0 \cdot 45$, thereby excluding the likelihood of important concordance (see above).

The pairs were then separated into the 17 HLA identical (sharing two haplotypes) and 28 nonidentical pairs (sharing one or no haplotype). The percentage congruence within the pairs (i.e., the proportion of the pairs within that group with both in the pair either positive or negative for a given variable) for the presence of nodules, Sjögren's syndrome, a positive latex or positive ANA titre was

Table 3 Concordance for positive latex and ANA tests

\begin{tabular}{|c|c|c|}
\hline & \multicolumn{2}{|c|}{ Number of pairs } \\
\hline & $\begin{array}{l}\text { Positive } \\
\text { latex }^{*}\end{array}$ & $\begin{array}{l}\text { Positive } \\
\text { ANA' }\end{array}$ \\
\hline Both members & 21 & 20 \\
\hline One member & 17 & 17 \\
\hline Neither member & 3 & 5 \\
\hline Kappa & $0 \cdot 00$ & +0.04 \\
\hline SE & $0 \cdot 19$ & $0 \cdot 17$ \\
\hline $95 \%$ Confidence & $-0 \cdot 38$ & $-0 \cdot 13$ \\
\hline interval & $+0 \cdot 38$ & $+0 \cdot 21$ \\
\hline p Value & NS & NS \\
\hline
\end{tabular}

*There were four pairs with one individual with no latex results. +There were three pairs with one individual with no ANA result. compared between the two HLA groups. Thus, for example, with nodules $29 \%$ of the HLA identical pairs had both members similar with respect to the presence or absence of nodules compared with $43 \%$ of the HLA non-identical pairs (p NS). Similarly, there were no differences for Sjögren's syndrome $(60 \% v 61 \%, \mathrm{p} \mathrm{NS})$ or ANA titre $(57 \% v 60 \%, \mathrm{p}$ NS). Conversely, the HLA identical pairs were more likely to be concordant for positive latex test $(75 \% v 58 \%)$, though this difference was not statistically significant. There was no greater similarity in age or calendar year of disease onset in the pairs with the shared haplotypes. A further analysis separating out those pairs with no shared haplotype from those sharing one and two did not alter the results.

\section{Discussion}

There was no evidence of greater disease heterogeneity between the families than between affected individuals within the families. Thus if there were aetiological differences between the families these differences were not reflected clinically or in the laboratory tests employed. More specifically the clinical features ascertained in this study were not related to the presumed shared genetic or environmental factors, or both, that led to disease susceptibility in affected family members.

The presence of HLA haplotype sharing between affected members did not alter the above conclusion. Thus HLA haplotype was not associated with disease heterogeneity in these families and was not associated with any specific disease subgroups. The results from the latex test may be an exception, but a larger study would be necessary to show whether or not affected members who shared HLA haplotypes were more likely to be congruent for a positive latex titre than other families with partial or no sharing.

There are three caveats in interpreting these data. Firstly, there might have been treatment differences between the affected members within a family; there is insufficient information to answer this. The presence of Sjögren's syndrome, nodules, ANA positivity, and the pattern of joint involvement (the variables used in this study) are less likely to be affected by treatment, however, than are variables indicating joint inflammation or functional ability. Secondly, these data were collected at only one time and therefore recorded only current and past disease expression. It might be, for example, that follow up would show more cases of Sjögren's syndrome or an altered pattern of joint involvement. It is unlikely, however, that the cross sectional approach selectively under-recorded the presence of disease attributes in the discordant as opposed to the negatively 
concordant pairs. In addition, the overall rate for nodules of $43 \%$ and seropositivity of $72 \%$ do not suggest underascertainment compared with standard descriptions of the disease. ${ }^{12}$ Thirdly, the analysis of the ' $n$ ' trios as ' $3 n$ ' pairs might, as stated earlier, bias the results towards greater concordance. In fact this potential bias would operate against the direction of the observed results and hence strengthen the conclusions on nonconcordance.

The failure to find homogeneity within families for each age or calendar year of onset merits attention. A similar age of onset within pairs would have supported a shared genetic susceptibility, whereas a similar calendar year of onset would have supported a shared environmental influence. The absence of either does not exclude a similar multifactorial inheritance within the sib pairs but does suggest that the cause of RA in these families is complex and hence unlikely to be explained by simple combinations of identified genetic and environmental factors. It is accepted that there might be error in the recalled date of onset, especially in those with disease of long duration. It is unlikely that such an error would either be selective in producing relatively greater discordance between pairs or numerically large enough to affect the results to any important extent.

In conclusion, genetic factors may have only a limited role in explaining disease heterogeneity in RA. Thus the identification of clinical subgroups is unlikely to be informative in unravelling the complex genetic background of RA. Finally, in view of these observations families with multiple cases of rheumatoid arthritis may not be a biased tool for investigating linkage between genetic markers and disease in the general population.
This investigation resulted from a family study of the immunogenetics of rheumatoid arthritis and was financially supported by the Arthritis and Rheumatism Council. We are grateful to Professor $\mathrm{H}$ Festenstein, the director of the family study, for his support and $\mathrm{Mr}$ $\mathrm{N}$ Gosnell, SRN who assisted in the examination and bleeding of the patients. We are also grateful for the support of our clinical colleagues, both at this and other units. for notifying us of the families used in this study.

\section{References}

1 Lawrence J S. Rheumatism in populations. London: Heinemann, 1977: 232-6.

2 Lawrence J S. Rheumatoid arthritis: nature or nurture. Ann Rheum Dis 1970; 29: 357-79.

3 Stastny P. Association of the B-cell alloantigen DR4 with or rheumatoid arthritis. $N$ Engl J Med 1978; 298: 868-9.

4 Panayi G S, Wooley P, Batchelor J R. Genetic basis of $\vec{\omega}$ rheumatoid disease: HLA antigens, disease manifestations and $\omega$ toxic reactions to drugs. $\mathrm{Br} \mathrm{Med} J$ 1978; ii: 1326-8.

5 McMichael A J, Sasazuki T, McDevitt H O, Payne R O. Increased frequency of HLA Cw3 and HLA Dw4 in rheumatoid arthritis. Arthritis Rheum 1977; 20: 1037-42.

6 Jaraquemada D, Pachoula-Papasteriadis C. Festenstein H, et al. HLA-D and DR determinants in rheumatoid arthritis. Transplant Proc 1979; 11: 1306.

7 Doubloug J, Forre O, Kasse E, et al. HLA antigens and rheumatoid arthritis. Association between HLA-DRw4 ang IgM rheumatoid factor production. Arthritis Rheum 198\%, 23: 309-13.

8 Ropes W. Diagnostic criteria for rheumatoid arthritis. $19 \overline{\overline{8}}$ revision by a committee of the American Rheumatism Associa tion. Ann Rheum Dis 1959; 18: 49.

9 Burns $T$ M, Calin $A$. The hand radiograph as a diagnostic discriminant between seropositive and seronegative rheumatoid arthritis: a controlled study. Ann Rheum Dis 1983; 42: 605-12.

10 Cohen J. A coefficient of agreement for nominal scales. Educ Psychol Measurement 1960; 20: 37-46.

11 Fleiss J L. Statistical methods for rates and proportions. 2nd ed. New York: Wiley, 1981: 232-3.

12 Thompson M. Rheumatoid arthritis. In: Hawkins C, Currey H L F, eds. Rheumatic diseases. Collected reports 1959-1983. London: Arthritis and Rheumatism Council, 1983: 11-4. 